\title{
Current-induced giant vortex and asymmetric vortex confinement in microstructured superconductors
}

\author{
X. H. Chao and B. Y. Zhu \\ National Laboratory for Superconductivity, Institute of Physics, and Beijing National Laboratory for Condensed Matter Physics, \\ Chinese Academy of Sciences, Beijing 100190, China
}

\author{
A. V. Silhanek and V. V. Moshchalkov \\ Institute for Nanoscale Physics and Chemistry (INPAC), Katholieke Universiteit Leuven, Celestijnenlaan 200D, B-3001 Leuven, Belgium
}

(Received 7 March 2009; revised manuscript received 5 June 2009; published 5 August 2009)

\begin{abstract}
Based on the time-dependent Ginzburg-Landau equations, we study numerically current-driven vortices in a micrometer size square type-II superconductor. We demonstrate that the applied current significantly influences the dynamics of the vortices entering the sample. Strikingly, we find that a giant vortex can be created by the current-assisted collision of two singly quantized vortices.
\end{abstract}

DOI: 10.1103/PhysRevB.80.054506

PACS number(s): 74.78.Na, 74.20.De, 74.81.-g

\section{INTRODUCTION}

The rapid development in nanotechnology during the last few decades has enormously facilitated the fabrication and investigation of submicrometer scale systems. Particular attention has been devoted to mesoscopic superconductors where the sample dimensions become comparable to the superconducting characteristic length scales. From the experimental point of view the most suitable materials for these studies are those with large coherence length typically obtained in conventional low $T_{c}$ superconductors such as Al.

In this low dimensionality limit, a plethora of novel phenomena has been revealed making this sort of systems a hot research topic in condensed-matter physics. The vortex distribution in small superconducting disks within London approximation was first calculated by Buzdin and Brison. ${ }^{1}$ Later on, using Ginzburg-Landau formalism, Palacios ${ }^{2}$ showed that vortices arrange themselves in shell structures with two consecutive states of different vorticity separated by a first-order transition. These results nicely agreed with the clear magnetization jumps detected by local Hall probes. ${ }^{3}$ More recently Baelus, Cabral, and Peeters ${ }^{4}$ studied the case of a large enough disk containing several shells of vortices. These authors demonstrated that contrary to small disks, two consecutive vortex configurations can be separated by a change in vorticity larger than one. More interestingly, a coexistence of a giant vortex state with multivortex state can be obtained in this case. Furthermore, a rich zoology of vortex configuration, including vortex shells following magic numbers, ${ }^{4,5}$ vortex-antivortex molecules, ${ }^{6}$ and giant vortices $^{7-15}$ in mesoscopic superconductors has been reported both theoretically and experimentally.

Despite the large number of studies of giant vortices in equilibrium conditions, little is known about their stability and evolution in mesoscopic superconductors under an applied current. This is a particular relevant issue since one of the most popular ways to determine the change in vorticity of mesoscopic superconductors is through electrotransport measurements. ${ }^{16,17}$ In this paper, we study the influence of a bias external current on the vortex dynamics in a square mesoscopic superconductor by solving numerically the time- dependent Ginzburg-Landau (TDGL) equations. The most interesting result is the unexpected formation of a giant vortex as a result of two single-quantized vortices collision. This giant vortex represents an unstable state which eventually splits in two single-quantum vortices.

\section{MODEL}

The vortex dynamics was investigated using the wellknown TDGL equations. ${ }^{18-23}$ In the Ginzburg-Landau regime, a superconductor is characterized by a complex order parameter $\psi$ with $|\psi|^{2}$ representing the local density of Cooper pairs $n_{s}$. The equations for the order parameter $\psi$, the vector potential $\mathbf{A}$, and the electric potential $\phi$ can be written $\mathrm{as}^{24}$

$$
\begin{aligned}
\frac{\hbar^{2}}{2 m_{s} D}\left(\frac{\partial}{\partial t}+\frac{i e_{s}}{\hbar} \phi\right) \psi+\frac{1}{2 m_{s}}\left(\frac{\hbar}{i} \nabla-\frac{e_{s}}{c} \mathbf{A}\right) \cdot\left(\frac{\hbar}{i} \nabla-\frac{e_{s}}{c} \mathbf{A}\right) \psi \\
-a|\psi|+b|\psi|^{2} \psi=0 \\
\nabla \times \nabla \times \mathbf{A}=-\frac{4 \pi \sigma}{c}\left(\frac{1}{c} \frac{\partial \mathbf{A}}{\partial t}+\nabla \phi\right)+\frac{4 \pi}{c} \mathbf{J}_{s}
\end{aligned}
$$

Here $e_{s}(=2 e)$ and $m_{s}$ are the effective charge and the effective mass of the Cooper pairs. $D$ is the diffusion coefficient and $\sigma$ is the conductivity. $a$ and $b$ are phenomenological parameters which depend on control variables such as the temperature. $\mathbf{J}_{s}=\frac{e_{s} \hbar}{2 i m_{s}}\left(\psi^{*} \nabla \psi-\psi \nabla \psi^{*}\right)-\frac{e_{s}^{2}}{m_{s} c}|\psi|^{2} \mathbf{A}$ is the supercurrent density. By setting $c=1$ and scaling the position $\mathbf{r}$ in units of $\xi(T)=\hbar / \sqrt{2 m|a|}$, the time $t$ in units of $\tau=\xi^{2} / D, \psi$ in units of $\sqrt{|a| / b}, \mathbf{A}$ in units of $A_{0}=\sqrt{2} \kappa H c \xi, \phi$ in units of $(\xi / \tau) A_{0}$, and $\sigma$ in units of $1 / 4 \pi \kappa^{2} D$, we obtain the dimensionless TDGL equations

$$
\left(\frac{\partial}{\partial t}+i \phi\right) \psi=(\nabla-i \mathbf{A})^{2} \psi+\psi-|\psi|^{2} \psi
$$




$$
\kappa^{2} \nabla \times \nabla \times \mathbf{A}=\operatorname{Im}\left[\psi^{*}(\nabla-i \mathbf{A}) \psi\right]+\sigma\left(-\nabla \phi-\frac{\partial \mathbf{A}}{\partial t}\right) .
$$

Here $\sigma=1$ and the magnetic field is always perpendicular to the sample. The TDGL equations are invariant under the gauge transformation by the formula in Ref. 24, i.e., $\psi^{\prime}$ $=\psi e^{i \kappa}, A^{\prime}=A+\nabla \chi$, and $\phi^{\prime}=\phi-\frac{\partial \chi}{\partial t}$, where the gauge $\chi$ is any function of space and time. We employ the normal $U-\psi$ method $^{22,24}$ in the finite-element regime to discretize Eqs. (3) and (4).

The applied current can be introduced via either the electric potential ${ }^{23} \phi$ in Eq. (3) or by imposing a magnetic field difference between the upper and lower boundaries. ${ }^{20,21} \mathrm{In}$ the present work, for simplicity we only implement the current effect by $\phi(x, y)=\gamma x$ in Eq. (3) but neglect the contribution of the constant term $\sigma \nabla \phi$ in Eq. (4), where $\gamma$ denotes the applied current density and $x$ is the coordinate along the $x$ direction. Thus, the transport current is always parallel to the $x$ axis. Actually, we have tested three different ways to mimic the current effect in the simulation system and all these methods bring no change to the main results on the vortex dynamics obtained in the present work.

The density of Gibbs free energy of the investigated system is given by

$$
\begin{aligned}
g= & g_{n 0}+\alpha|\psi|^{2}+\frac{\beta}{2}|\psi|^{4}+\frac{1}{2 m^{*}}\left|\left(\frac{\hbar}{i} \nabla-\frac{e^{*}}{c} \mathbf{A}\right) \psi\right|^{2}+\frac{h^{2}}{8 \pi} \\
& -\frac{\mathbf{h} \cdot \mathbf{H}}{4 \pi},
\end{aligned}
$$

where $h$ is the magnetic induction. If the magnetic field $H$ is normalized by $H_{c 2}=\sqrt{2} \kappa H_{c}$, we obtain the dimensionless energy expression

$$
E=g-g_{n 0}=-|\psi|^{2}+\frac{1}{2}|\psi|^{4}+\left|\left(\frac{\nabla}{i}-\mathbf{A}\right) \psi\right|^{2}+\kappa^{2} \mathbf{h}(\mathbf{h}-2 \mathbf{H}) .
$$

The magnetization of the system is given by $\mathbf{M}$ $=1 / 4 \pi(\mathbf{h}-\mathbf{H})$. Here we want to mention that thermal fluctuations may induce an asymmetric vortex entrance into the square sample. However, in order to reveal clearly the influence of the applied current on the vortex distribution and the dynamics, we neglect the thermal fluctuation term in the simulation accordingly, which does not change the intrinsic properties revealed in the present study.

The boundary conditions for the order parameter depend on the physical nature of the boundary. ${ }^{25,26}$ For the sample borders perpendicular to the $y$ direction, we assume that the perpendicular component of the superconducting current is equal to zero at the surface $\left(\left.J_{s}\right|_{n}=0\right)$, where the suffix $n$ denotes the direction normal to the boundary (i.e., $\left.\left.(\nabla-i \mathbf{A})\right|_{n} \psi=0\right)$. In the $x$ direction we introduce metalsuperconductor boundary condition. For the order parameter $\psi$, it can be written as $\left.(\nabla-i \mathbf{A})\right|_{n} \psi=-\frac{\psi}{b}$, where $b$ is a real constant. ${ }^{25,27,28}$ In this work, for simplicity, we present results assuming $b=20$, although other values of the parameter $b$ show no significant differences. The vector potential $\mathbf{A}$ at

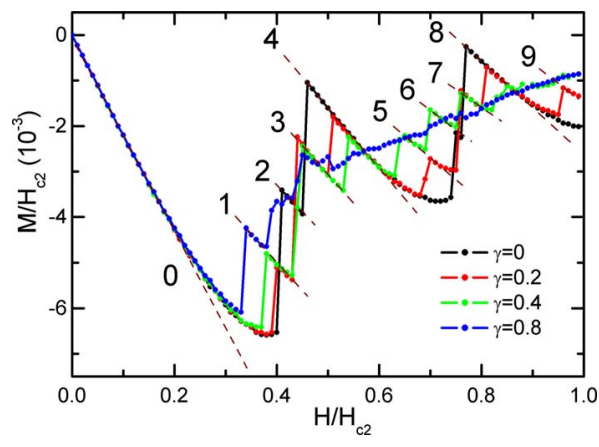

FIG. 1. (Color online) Magnetization as a function of the external magnetic field for different current densities $\gamma=0,0.2,0.4$, and 0.8 , respectively. The arabic numerals indicate the total vorticities of the system.

the boundary is determined by the external magnetic field $\mathbf{H}$, with $\nabla \times \mathbf{A}=\mathbf{H}$. Since the external magnetic field is always parallel to the $z$ axis we take $\mathbf{H} \equiv H \hat{\mathbf{z}}$.

As a model system, we consider a conventional superconductor infinite in the $z$ direction with $\kappa=2.6$ and lateral size $9.7 \xi \times 9.7 \xi$. The mesh used in our simulation is chosen as $\Delta x=\Delta y=0.1 \xi$ and the time step is chosen as $\Delta t=0.00036 \tau$ with $\tau \equiv 1$.

\section{SIMULATION AND DISCUSSION}

In Fig. 1, we show the average magnetization as a function of the applied magnetic field for various current densities $\gamma=0,0.2,0.4$, and 0.8 , respectively. When the current is applied along the $x$ direction, it gives rise to a force on the vortices along the $y$ direction. This lateral force keeps the vortices moving toward one side of the square. This process will be continuous if the driving force is large enough, that is, vortices are nucleated at the top of the square while others scape through the bottom of the square. Therefore, for a certain vorticity $L$ and applied field $H$, we indicate in Fig. 1 the magnetization value, averaged in $5 \times 10^{5}$ run steps in order to ensure a stable vortex flow state in the system. For $\gamma=0$, obviously the average value of $M$ is corresponding to the stationary state. The initial increase in the magnetic field is accompanied by a strong diamagnetic response corresponding to the superconducting Meissner state. Due to the different boundary conditions along $x$ and $y$ axes, two vortices enter the sample from the opposite edges parallel to the $y$ axis at $H / H_{c 2}=0.41$. For $H / H_{c 2}=0.46$, four vortices nucleate and penetrate into the square. ${ }^{29,30}$ Further increasing the field, two more jumps occur at $H / H_{c 2}=0.75$ and 0.77 , where six and eight vortices are nucleated in the system.

A completely different situation appears when an external current is applied into the system. Indeed, for $\gamma=0.2$ and 0.4 , the magnetization curves exhibit more branches, each of them associated with a change in the total vorticity $L$ or the quantum vortex number in the system. This result suggests that the applied current breaks the square symmetry imposed by the sample geometry by introducing a tilted potential. As a consequence of this symmetry transformation, vortices can enter the sample one by one as evidenced by the jumps in 

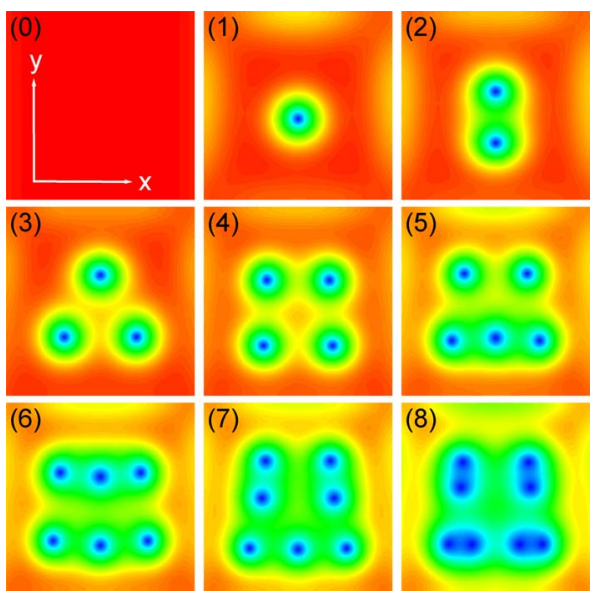

(8)

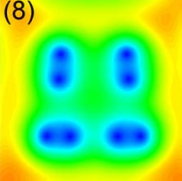

FIG. 2. (Color online) Contour plots of $|\psi|$ at $\gamma=0.4$ for different fields $H / H_{c 2}=0,0.38,0.44,0.45,0.54,0.65,0.7,0.76$, and 0.85 , respectively. The dots represent the vortex core positions in the system with low $|\psi|$ value. The index numbers (0)-(8) in each plot also present the total vorticity $L$ in the system.

$M(H)$ shown in Fig. 1 for $\gamma=0.4$. It is interesting to note that for $\gamma=0.2$, the system remains in the branches of $L=4$ or 8 over a wider magnetic field range with respect to the other branches. This effect results from the fact that these branches correspond to more symmetric distributions of the vortices in the square system. For $\gamma=0.8$, only few branches of small $L$ values are still discernible in the $M(H)$ curve whereas the discrete steplike structure disappears for large magnetic fields $\left(H / H_{c 2}>0.5\right)$. This behavior can be understood as follows, when the sample is exposed to a large current, the vortices flow in the sample in a continuous fashion preventing the formation of stable configurations which eventually give rise to the step structure. Notice also that increasing the current density $\gamma$ from 0 to 0.8 , the lower critical magnetic field $H_{c 1}$ is reduced, as expected.

Figure 2 shows the contour plots of the magnitude of the order parameter $|\Psi|$ for the final steady states in the sequence of branches at $\gamma=0.4$ as seen in Fig. 1. Increasing the field leads to an increase in the vortex number one by one. It is important to emphasize that this behavior is in sharp contrast to that obtained in samples without current, where a singlevortex entry is prohibited. It has been extensively demonstrated that in a large system the vortices tend to form a triangular Abrikosov lattice. This scenario can change dramatically in small samples where the boundary effects confine the equilibrium vortex configurations. ${ }^{6,29}$ Figure 2 shows that a more complex vortex configuration can be obtained by combining a mesoscopic sample with an external bias current.

In Fig. 3, we show a series of snapshots of both the magnitude and the phase of the order parameter for the currentdriven vortices in the square sample. In Fig. 3(a) and 1, three vortices form a triangular lattice with an axial symmetrical structure along $y$ axis. Because of the Lorentz force induced by the applied current, vortices move slowly toward the negative $y$ direction. In Fig. 3(b) and 1, a new vortex enters the sample and runs into the upper vortex of the triangle. This collision results from the fact that the central vortex moves slower than the new intruded one as a consequence of the vortex-vortex interaction. Figure 3(c) and 1 shows that the incoming vortex collides with the central one and they merge into a giant vortex with $L=2$. This remarkable coexistence of a giant vortex with two satellite single-quantum $\Phi_{0}$ vortices below, forming a noncentral symmetric structure, is different from other giant vortex matter obtained in mesoscopic samples without external current. ${ }^{9}$ As it is shown in the next images [Figs. 3(d), 1, 3(e), and 1], this giant $2 \Phi_{0}$ vortex is not stable and eventually splits in two $\Phi_{0}$ vortices due to the strong repulsive interaction between them.

Figures 3(a), 2, 3, and 2 show the contour plots of the phase of the order parameter corresponding to the snapshots shown in Figs. 3(a), 1-3, and 1. An integration path around the superconductor shows that the phase changes $3 \times 2 \pi$ in Fig. 3(a) and 2 and $4 \times 2 \pi$ in Figs. 3(b), 2, 3, and 2. In addition, around isolated vortices, as seen in Figs. 3(a), 2, $3(\mathrm{~b}), 2,3(\mathrm{~d})$, and 2 , the phase changes by $2 \pi$, whereas in Fig. 3 (c) and 2 it changes by $4 \pi$ around the central giant vortex. This result provides unambiguous and compelling evidence of the formation of a giant vortex state.

In order to understand the process of nucleation and annihilation of the giant vortex, we calculated the corresponding free energy, magnetization, and vorticity for the whole
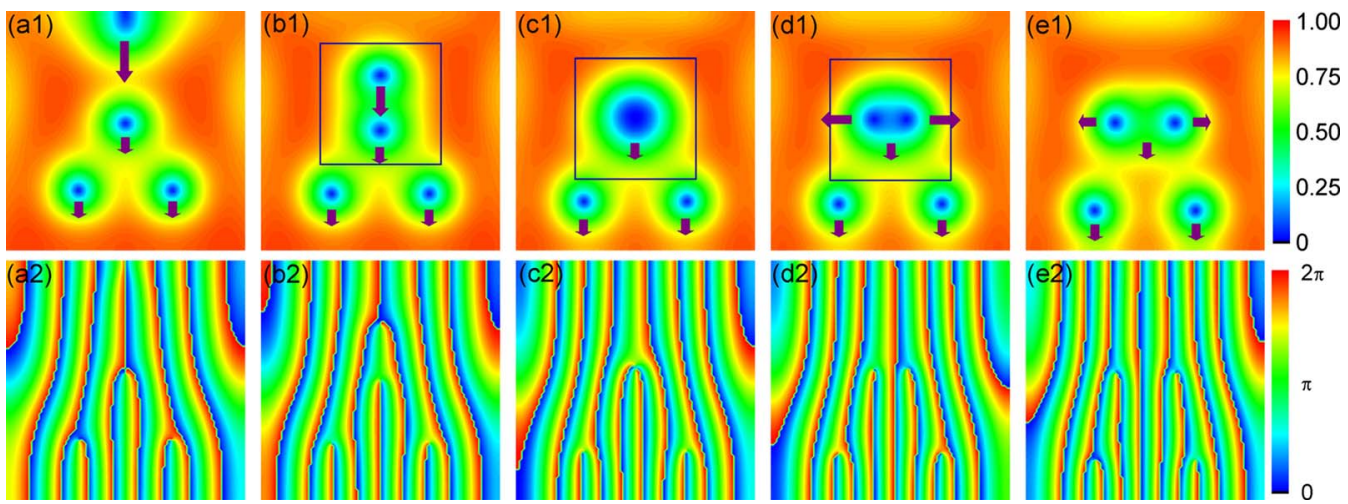

FIG. 3. (Color online) (a1)-(e1) and (a2)-(e2) are contour plots of the distribution of the magnitude of the order parameter $|\psi|$ and their corresponding phase $\varphi$ at $H / H_{c 2}=0.5$ with the applied current density $\gamma=0.8$ along $x$ direction at different time: (a1) and (a2): 77.76; (b1) and (b2): 86.04; (c1) and (c2): 95.688; (d1) and (d2): 98.28; and (e1) and (e2): 104.4. The arrows illustrate the direction and the speed of the vortex motion. 

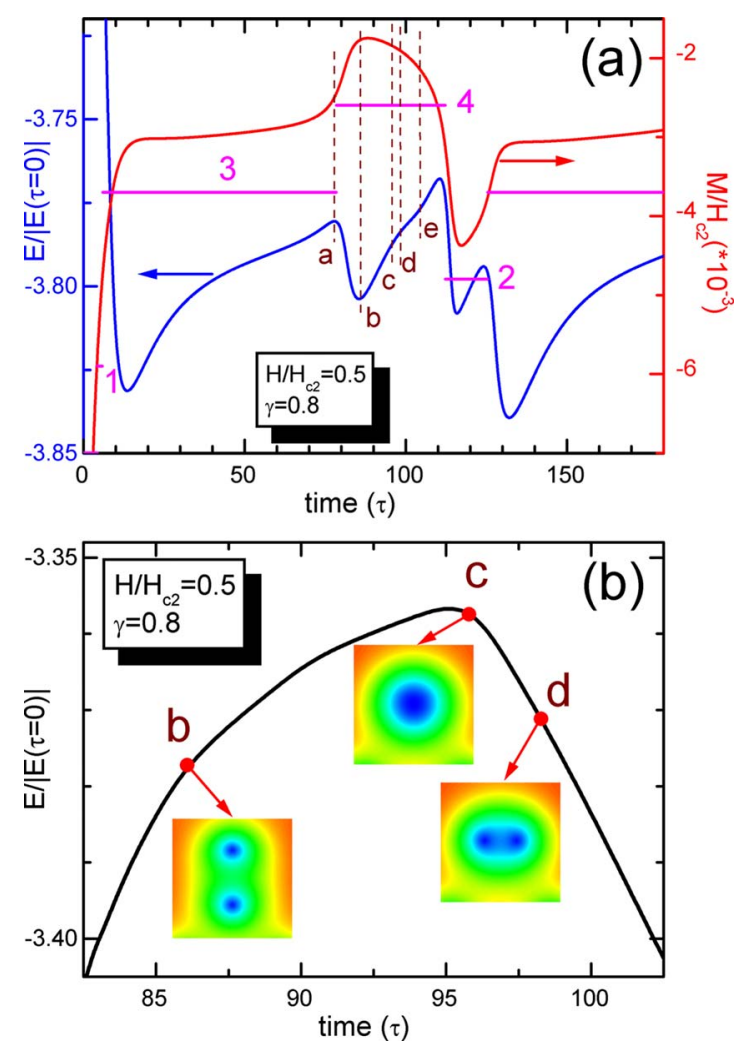

FIG. 4. (Color online) (a) Time evolution of free-energy (blue curve), magnetization (red curve), and vorticity (magenta segment lines) of the square superconducting system with $\gamma=0.8$ and $H / H_{c 2}=0.5$. The dashed vertical lines a-e guide the eyes for the evolution corresponding to the snapshots shown in Fig. 3. (b) Time evolution of free energy in the designated patches with the same size for the process of the giant vortex formation. The insets show the snapshots at point b, c, and d, which have been framed in Figs. 3 (b), 1-3, and 1 .

system as a function of time. The results are summarized in Fig. 4(a). The free energy exhibits a series of cusps as a function of time each of which corresponding to a change in vorticity in the system. Furthermore, the magnetization curve is in agreement with the vorticities of the superconducting system, which represents the diamagnetic variation in the sample.

At the point $a$ in Fig. 4(a), the magnetic signal increases rapidly and the free energy reaches a local maximum corresponding to the nucleation of a new vortex in the boundary region [Fig. 3(a) and 1]. After the entrance of the new incoming vortex, the whole system composed by the four vortices undergoes a relaxation process reflected in the decrease in the free energy till point $b$. Later on, as anticipated in Figs. $3(\mathrm{c}), 1,3(\mathrm{c})$, and 2 , two $\Phi_{0}$ vortices collide and merge into a giant vortex at point $c$. This event manifests itself as an increase in the free energy after point $b$ and a kink structure around points $c$ and $d$ as seen in Fig. 4(a). After this, the free energy continues to increase and reaches a peak value corresponding to the sharp drop of the magnetization at $t \approx 112$, where two vortices are removed from the sample by the applied current.

In general, the process of vortex collision and formation of a giant vortex is difficult to identify in the free energy.

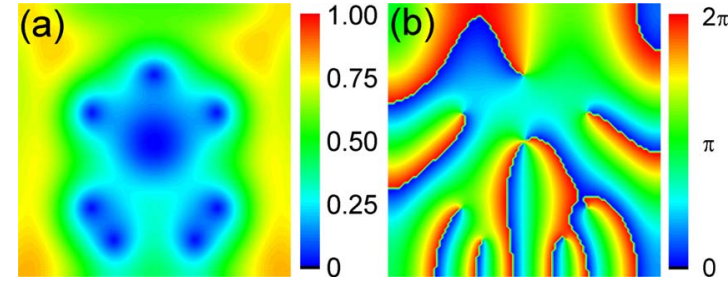

FIG. 5. (Color online) Contour plots of (a) $|\psi|$ and (b) $\varphi$ of the order parameter with $H / H_{c 2}=0.9$ and $\gamma=0.8$ at certain time in the simulation.

This is due to its small contribution to the total energy which is dominated by the self-energy of the vortices. The global free-energy maximum or the saddle point always indicates a change in the vortex number during the vortex flow in the sample. Since the collision process happens in the nonequilibrium vortex flow state, the signal response of the evolution of the collision process is always smaller than that of the vortex nucleation or vortex annihilation at the edges in the contribution of the global free energy as seen in Fig. 4(a). Therefore, from the global free energy is hard to disclose the vortex-collision process as well as the nucleation and the annihilation of the giant vortex in the multivortex system with changeable vorticities. Additionally, since the total vorticity of the system retains $L=4$ from $t \approx 78$ to 112 , the process of nucleation and annihilation of the giant vortex shows no features in the magnetization curve. In order to understand the transition between the giant vortex and the singlequantum vortices, in Fig. 4(b), we show the evolution of the free-energy zooming in the time window where the process of giant vortex formation occurs. The calculation demonstrates clearly that the maximum free energy of the designated area coincides with the giant vortex state. Therefore, we believe that the local free energy in the collision area by neglecting the irrelevant events in the whole system can properly characterize the nucleation and the annihilation process of the giant vortex transformation. We would like to mention that the dynamic origin of formation of a giant vortex is substantially different than the static case, $, 9,29,31$ where the total vorticity can be revealed exactly by the free energy. We also want to point out that the vortex collision and the giant vortex always appear in a very short time scale in the nonequilibrium state in the perfect system. The possible way to extend the lifetime of the giant vortex is to introduce the pinning defect in the system, which may make it easier to observe the giant vortex matter experimentally.

In what follows, we will demonstrate that the formation of giant vortices becomes easier at high magnetic fields. In this limit, more $\Phi_{0}$ vortices flow inside the sample at the same time and the probability of vortex collision increases. In Figs. 5(a) and 5(b), we show the contour plots of both the magnitude and the phase of the order parameter for the vortex dynamics in the current driven system at $H / H_{c 2}=0.9$. One can see clearly the coexistence of single-quantized vortices and a giant vortex in the system. Interestingly, for high magnetic fields, giant vortices can bear more than two flux quanta. Additionally, we have carried out the simulations for different sample sizes and $\kappa$ values to determine the relevance of these parameters in the formation of giant vortices. 
In a large square sample, we mostly obtain a moving lattice of single quantized $\Phi_{0}$ vortices with the applied current. This finding suggests that the limited size of the sample is the most crucial factor for the giant vortex formation, in similar fashion to the giant vortex formation in a system without current. ${ }^{9,29,31}$ We have also checked that the collision event and the subsequent giant vortex formation occurs in a wide range of $\kappa$ values as long as the external magnetic field and the current density in the system are properly tuned.

\section{SUMMARY}

We have studied numerically the current-driven vortex dynamics in a square mesoscopic superconductor by TDGL equations. We showed that the applied current can break the symmetry imposed by the sample geometry and significantly influence the vortex penetration and distribution in the sample. Our simulations show a series of asymmetric configurations of the vortex entries under the bias current, which are quite different from the symmetry distribution obtained without the current. ${ }^{29}$ Although the occurrence of a singlevortex entry in a symmetric square sample can be excited by the thermal fluctuation which is neglected here, ${ }^{14,15,29}$ the configuration and the directional flow of the vortex lattice inside the sample cannot be duplicated. In addition, we have found clear giant vortex formation through a collision involving two individual vortices. Clear evolution of the vortex configurations for the nucleation and the annihilation of the giant vortex has been revealed when the system is exposed to the current. We propose that the local free energy characterizes well the process of the nucleation and annihilation of the giant vortex. We also want to emphasize that the surface barrier plays a crucial role for the giant vortex formation in the collision process, which not only accelerates the new born vortex to rush into the central area but also decelerates or even stops the vortex to escape from the sample due to the driving current. Our study reveals a method for the giant vortex formation in the mesoscopic superconductor and it is beneficial for the design of the current-driven vortex device based on the microstructured superconductors, such as the quantum-fluxon rectifiers, vortex pumps, diodes, or lenses. ${ }^{32}$

\section{ACKNOWLEDGMENTS}

We acknowledge the support from the MOST, NSF, SRF for ROCS, SEM, and the BIL Flanders-China Projects of China. This work was also supported by Methusalem Funding of the Flemish government, FWO-Vlaanderen, and the Belgian Inter-University Attraction Poles IAP Programmes. A.V.S. is grateful for the support from the FWO-Vlaanderen.
${ }^{1}$ A. I. Buzdin and J. P. Brison, Phys. Lett. A 196, 267 (1994).

${ }^{2}$ J. J. Palacios, Phys. Rev. B 58, R5948 (1998).

${ }^{3}$ A. K. Geim, I. V. Grigorieva, S. V. Dubonos, J. G. S. Lok, J. C. Maan, A. E. Filippov, and F. M. Peeters, Nature (London) 390, 259 (1997)

${ }^{4}$ B. J. Baelus, L. R. E. Cabral, and F. M. Peeters, Phys. Rev. B 69 , 064506 (2004).

${ }^{5}$ H. J. Zhao, V. R. Misko, F. M. Peeters, V. Oboznov, S. V. Dubonos, and I. V. Grigorieva, Phys. Rev. B 78, 104517 (2008); V. R. Misko, B. Xu, and F. M. Peeters, ibid. 76, 024516 (2007); V. R. Misko and F. M. Peeters, ibid. 74, 174507 (2006).

${ }^{6}$ L. F. Chibotaru, A. Ceulemans, V. Bruyndoncx, and V. V. Moshchalkov, Nature (London) 408, 833 (2000); Phys. Rev. Lett. 86, 1323 (2001); V. R. Misko, V. M. Fomin, J. T. Devreese, and V. V. Moshchalkov, ibid. 90, 147003 (2003).

${ }^{7}$ B. J. Baelus, A. Kanda, N. Shimizu, K. Tadano, Y. Ootuka, K. Kadowaki, and F. M. Peeters, Phys. Rev. B 73, 024514 (2006); B. J. Baelus, A. Kanda, F. M. Peeters, Y. Ootuka, and K. Kadowaki, ibid. 71, 140502(R) (2005); A. K. Geim, S. V. Dubonos, J. J. Palacios, I. V. Grigorieva, M. Henini, and J. J. Schermer, Phys. Rev. Lett. 85, 1528 (2000); P. S. Deo, V. A. Schweigert, F. M. Peeters, and A. K. Geim, ibid. 79, 4653 (1997).

${ }^{8}$ A. Kanda, B. J. Baelus, F. M. Peeters, K. Kadowaki, and Y. Ootuka, Phys. Rev. Lett. 93, 257002 (2004).

${ }^{9}$ B. J. Baelus and F. M. Peeters, Phys. Rev. B 65, 104515 (2002).

${ }^{10}$ V. A. Schweigert, F. M. Peeters, and P. S. Deo, Phys. Rev. Lett. 81, 2783 (1998).

${ }^{11}$ E. Sardella, A. L. Malvezzi, P. N. Lisboa-Filho, and W. A. Ortiz, Phys. Rev. B 74, 014512 (2006).

${ }^{12}$ V. Bruyndoncx, J. G. Rodrigo, T. Puig, L. Van Look, V. V. Mosh- chalkov, and R. Jonckheere, Phys. Rev. B 60, 4285 (1999).

${ }^{13}$ I. V. Grigorieva, W. Escoffier, V. R. Misko, B. J. Baelus, F. M. Peeters, L. Y. Vinnikov, and S. V. Dubonos, Phys. Rev. Lett. 99, 147003 (2007).

${ }^{14}$ T. Nishio, S. Okayasu, J. Suzuki, and K. Kadowaki, Physica C 379, 412 (2004)

${ }^{15}$ S. Okayasu, T. Nishio, Y. Hata, J. Suzuki, I. Kakeya, K. Kadowaki, and V. V. Moshchalkov, IEEE Trans. Appl. Supercond. 15, 696 (2005).

${ }^{16}$ N. Schildermans, A. Yu. Aladyshkin, A. V. Silhanek, J. Van de Vondel, and V. V. Moshchalkov, Phys. Rev. B 77, 214519 (2008).

${ }^{17}$ M. Morelle, V. Bruyndoncx, R. Jonckheere, and V. V. Moshchalkov, Phys. Rev. B 64, 064516 (2001)

${ }^{18}$ C. R. Hu and R. S. Thompson, Phys. Rev. B 6, 110 (1972).

${ }^{19}$ T. Winiecki and C. S. Adams, J. Comput. Phys. 179, 127 (2002).

${ }^{20}$ M. Machida and H. Kaburaki, Phys. Rev. Lett. 71, 3206 (1993).

${ }^{21}$ D. Y. Vodolazov and F. M. Peeters, Phys. Rev. B 76, 014521 (2007).

${ }^{22}$ R. Kato, Y. Enomoto, and S. Maekawa, Phys. Rev. B 44, 6916 (1991); 47, 8016 (1993).

${ }^{23}$ Q. Du, M. D. Gunzburger, and J. S. Peterson, SIAM Rev. 34, 54 (1992); Q. Du, Contemp. Math. 329, 105 (2003).

${ }^{24}$ W. D. Gropp, H. G. Kaper, G. L. Leaf, D. M. Levine, M. Palumbo, and V. M. Vinokur, J. Comput. Phys. 123, 254 (1996).

${ }^{25}$ P. G. de Gennes, Superconductivity of Metals and Alloys (Adison-Wesley, New York, 1989).

${ }^{26}$ A. D. Hernández and D. Domínguez, Phys. Rev. B 65, 144529 (2002).

${ }^{27}$ E. A. Andryushin, V. L. Ginzburg, and A. P. Silin, Usp. Fiz. 
Nauk 163, 105 (1993)

${ }^{28}$ M. Tinkham, Introduction to Superconductivity (Dover, New York, 2004).

${ }^{29}$ S. Kim, C.-R. Hu, and M. J. Andrews, Phys. Rev. B 69, 094521 (2004).

${ }^{30}$ M. Morelle, J. Bekaert, and V. V. Moshchalkov, Phys. Rev. B
70, 094503 (2004).

${ }^{31}$ D. S. Golubović, M. V. Milosević, F. M. Peeters, and V. V. Moshchalkov, Phys. Rev. B 71 180502(R) (2005).

${ }^{32}$ B. Y. Zhu, F. Marchesoni, V. V. Moshchalkov, and F. Nori, Phys. Rev. B 68, 014514 (2003); B. Y. Zhu, F. Marchesoni, and F. Nori, Phys. Rev. Lett. 92, 180602 (2004). 